\title{
Laser Interferometry Analysis of Ciprofloxacin Diffusion through Pseudomonas aeruginosa Biofilm
}

\section{Arabski $\mathbf{M}^{1 *}$, Wạsik $\mathrm{S}^{2}$ and Drulis-Kawa $\mathrm{Z}^{3}$}

${ }^{1}$ Department of Microbiology, Institute of Biology, Jan Kochanowski University in Kielce, Świętokrzyska 15, 25-406, Kielce, Poland ${ }^{2}$ Department of Molecular Physics, Institute of Physics, Jan Kochanowski University, Swiętokrzyska 15, 25-406, Kielce, Poland ${ }^{3}$ Department of Pathogen Biology and Immunology, University of Wroclaw, Przybyszewskiego 63/77, 51-148 Wroclaw, Poland

\begin{abstract}
In this study the novel application of laser interferometry method in quantitative analysis of drugs distributions in bacterial biofilms is presented. Ciprofloxacin diffusion through $P$. aeruginosa PAO1 biofilm was used as a model system. It was determined that $0.759 \mu \mathrm{mol}(25.32 \%)$ of ciprofloxacin was transported through biofilm after $2400 \mathrm{~s}$ from initial amount of $3 \mu \mathrm{mol}$. Additionally, laser interferometry method was used to calculate the amount (mol) of drug accumulated in the biofilm formed on nucleopore membrane. We observed that the amount of ciprofloxacin into biofilm is $0.366 \mu \mathrm{mol}(12.2 \%)$ after $2400 \mathrm{~s}$. These results were in accordance with measurements obtained by standard cultivation methods.
\end{abstract}

In conclusion, laser interferometry technique might be useful tool in real time calculation of drug concentration in bacterial biofilm as well as its transport through that structure. From clinical point of view, this important information might be used in modeling of antibiotics distribution in correlation with their biological effects on bacterial biofilms.

Keywords: Biofilm; Pseudomonas; Diffusion; Laser interferometry

\section{Introduction}

Bacterial biofilm is a surface attached form of unicellular microorganisms that come together to form a community and encased in an exopolysaccharide (EPS) [1]. Biofilm formation is a one of the major causes of failure in antimicrobial therapies, like antibiotic treatment in persistent bacterial infections. The EPS shield bacteria from opsonization and phagocytosis [2,3]. Moreover, bacteria in biofilms are less susceptible to antibiotics than their planktonic counterparts $[2,4]$. This effect might be explained by limitation of antibiotic penetration into biofilm [2]. The surface layers of biofilm are exposed to a lethal dose of antibiotic and these diffusion barriers protect against transport of the antibiotic dipper into the structure [2]. The resistance of biofilm forming bacterial cells against antibiotics might be conditioned by physiological limitations of microorganisms that exist in a recalcitrant phenotypic state in an altered chemical microenvironment within the biofilm [2].

Nowadays, the research methodology of antibiotic penetration analysis into bacterial biofilms is based on bioassay analysis [2], analysis of antibiotic penetration rates through the biofilm formed on cell culture inserts [5] or analysis of IR bands of the biofilm in regions of spectrum associated with nucleic acids in the presence of antibiotics (ATR/FT-IR) [6].

In the present work, we propose a new method in quantitative analysis of antibiotic diffusion through bacterial biofilm; laser interferometry. In our previously studies, this technique was successfully used in the measurements of Proteus lipopolisaccharides (LPS) interactions with colistin [7], chitosan [8], saponin [9], antibiotics and liposomes diffusion $[10,11]$. In this study, the application of laser interferometry technique is presented in quantitative analysis of ciprofloxacin distributions in Pseudomonas aeruginosa biofilm. The aminoglycosides (tobramycin and gentamycin) as well as fluoroquinolones like ciprofloxacin are the most commonly used antibiotics in the treatment of $P$. aeruginosa infections. Our previous studies indicated that ciprofloxacin was more efficient in inhibition/eradication of biofilm structure in comparison to gentamycin [12]. Therefore, laser interferometry method was utilized in presented paper to evaluate the role of ciprofloxacin distribution in biofilm structure in bacteria eradication process.

\section{Material and Methods}

\section{Culture conditions, membrane and chemicals}

P. aerugianosa $\mathrm{PAO} 1$ was cultivated under aerobic conditions in Luria-Bertani medium at $37^{\circ} \mathrm{C}$. Polymeric nuclear track membrane (nucleopore) with pores diameter $0.9 \mu \mathrm{m}$ was purchased from Joint Institute for Nuclear Research in Dubna, Russia. Ciprofloxacin was purchased from Krka (Nove Mesto, Slovenia).

\section{CV staining of $P$. aeruginosa biofilm}

Biofilm of $P$. aeruginosa $\mathrm{PAO} 1$ was formed at $37^{\circ} \mathrm{C}$ in $\mathrm{LB}$ (LuriaBertani) medium for $96 \mathrm{~h}$ in stationary conditions in (i) NUNC MaxiSorp microtitre plates for analysis of antibacterial effect of ciprofloxacin on $P$. aeruginosa biofilm or (ii) on sterile nucleopore membrane with pores diameter of $0.9 \mu \mathrm{m}$, as element of membrane system from laser interferometry equipment. The microtitre plates or nucleopore membranes with formed mature biofilm were washed 3 times by $300 \mu \mathrm{l}$ or $2 \mathrm{ml}$ of $0.9 \% \mathrm{NaCl}$ solution, respectively and were stained by crystal violet $(0.4 \%)$ for $15 \mathrm{~min}$. After staining the probes were washed in the same conditions as above and absorbance was measured with Microplate Reader TECAN Infinite 200 PRO (Tecan Group Ltd., Switzerland) at $531 \mathrm{~nm}$ (microtitre plates). The \%

*Corresponding author: Arabski M, Department of Microbiology, Institute of Biology, Jan Kochanowski University in Kielce, Świętokrzyska 15, 25-406, Kielce, Poland, Tel./Fax: (+48) 41349 6307; E-mail: arabski@ujk.edu.pl

Received January 28, 2013; Accepted March 27, 2013; Published March 31 2013

Citation: Arabski M, Wąsik S, Drulis-Kawa Z (2013) Laser Interferometry Analysis of Ciprofloxacin Diffusion through Pseudomonas aeruginosa Biofilm. Clin Microbial 2: 105. doi:10.4172/2327-5073.1000105

Copyright: @ 2013 Arabski M, et al. This is an open-access article distributed unde the terms of the Creative Commons Attribution License, which permits unrestricted use, distribution, and reproduction in any medium, provided the original author and source are credited. 
degree of nucleopore covering by PAO1 biofilm was calculated using image software (NIMH, USA) [7,13,14]. The membranes image were converted to grey-scale digital images with a CanoScan Lide 60 scanner (300 dpi resolution) and saved in a tiff file format. The mean of the greys (value 1 denote black color and value 256 denote white color) from three membrane images was used to determine the relative greyscale density of one treatment.

\section{Antibacterial effect of ciprofloxacin on P. aeruginosa biofilm}

The P. aeruginosa PAO1 biofilm was formed in NUNC MaxiSorp microtitre U-bottom plates for $72 \mathrm{~h}$ at $37^{\circ} \mathrm{C}$ in $\mathrm{LB}$ medium. Ciprofloxacin was derived from stock solution $(10 \mathrm{mg} / \mathrm{ml})$ and added to the $P$. aeruginosa PAO1 biofilm (after $72 \mathrm{~h}$ incubation at $37^{\circ} \mathrm{C}$ in LB medium and (washed 3 times by sterile medium) to give a final concentration in the range of $0.75-12 \mu \mathrm{mol} / \mathrm{ml}$ in the wells. The plates were incubated at $37^{\circ} \mathrm{C}$ for $2400 \mathrm{~s}$ (time of laser interferometry analysis). After incubation the wells were washed 3 times by $300 \mu$ or 2 $\mathrm{ml} 0.9 \% \mathrm{NaCl}$ and stained by crystal violet $(0.4 \%)$ for $15 \mathrm{~min}$. After the staining the probes were washed in the same conditions as above and absorbance was measured with Microplate Reader TECAN Infinite 200 PRO (Tecan Group Ltd., Switzerland) at $531 \mathrm{~nm}$.

\section{Laser interferometry}

The amount of ciprofloxacin, $N(t)$, which diffuses in time $t$ from a solution to water was calculated by integrating the concentration profile according to:

$$
N(t)=s \int_{0}^{\delta} C_{1}(x, t) d x,
$$

where $C_{1}(x, t)$ denotes the concentration of ciprofloxacin at a point situated at a distance $\mathrm{x}$ from the biofilm-growth medium (LB) interface, $S$ the surface of the biofilm-growth medium interface $\left(S=35 \times 10^{-6} \mathrm{~m}^{2}\right)$, and $\delta$ the concentration boundary layer (CBL) thickness.

The values $\mathrm{C}_{1}(\mathrm{x}, \mathrm{t})$ and $\delta$ were determined experimentally using interferograms (Figure 1B) obtained by laser interferometry system. The computer program used to analyse these images allows ascertaining the concentration profiles and CBL thicknesses. The interferograms, which appear due to the interference of two laser beams, are determined by the refraction coefficient of the solute, which in turn depends on the concentration of the substance. When the solute is uniform, the interference fringes are straight and they bend when a concentration gradient appears. The concentration profile $\mathrm{C}(\mathrm{x}, \mathrm{t})$ is determined by the deviation of the fringes from a straight course. Since the concentration $\mathrm{C}$ and the refraction coefficient are assumed to be linear, we have:

$$
C(x, t)=C_{0}+a \frac{\lambda d(x, t)}{h f},
$$

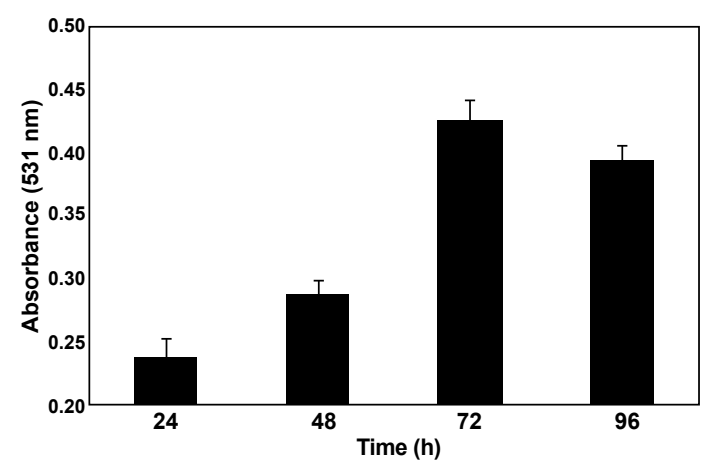

Figure 1: Analysis of $P$. aeruginosa PAO1 biofilm formation for $24-96 \mathrm{~h}$ at $37^{\circ} \mathrm{C}$ in LB medium determined by crystal violet staining. where $C_{0}$ is the initial substance concentration, $a$ the proportionality constant between the concentration and the refraction index $(a=6.60 \times$ $10^{3} \mathrm{~mol} / \mathrm{m}^{3}$ for the ciprofloxacin aqueous solution), $\lambda$ the wavelength of the laser light, $h$ the distance between the fringes in the field where they are straight lines, and $f$ the thickness of the solution layer in the measurement cuvette. The CBL thickness $\delta$ was defined as the distance from the biofilm-LB medium interface to the point at which the deviation $d$ of the interference fringe from its straight line run is $10 \%$ of the fringe thickness. By recording the interferograms at a given time interval one can reconstruct the concentration profiles at different times. Such profiles were used to calculate the membrane permeability coefficient.

The membrane system under study consists of two glass cuvettes (internal dimensions: $70 \mathrm{~mm}$ high, $10 \mathrm{~mm}$ wide, optical path length: $7 \mathrm{~mm}$ ) separated by the horizontally located nucleopore membrane (place of biofilm formation). The cuvettes are made with optical glass of high uniformity. After formation of PAO1 biofilm on nucleopore membrane for $72 \mathrm{~h}$ at $37^{\circ} \mathrm{C}$ and 3 times washing by sterile medium, the upper cuvette held pure LB medium $(1 \mathrm{ml})$, while the lower one was filled with ciprofloxacin at $3 \mu \mathrm{mol} / \mathrm{ml}$ concentration (in $1 \mathrm{ml}$ of LB medium). We used sterille membrane without biofilm as control. Experiments were performed at a temperature of $37 \mathrm{C}$.

The interferograms were recorded from 120 to $2400 \mathrm{~s}$ (stationary phase of diffusion detected by laser interferometry) with a time interval of $\Delta t=120 \mathrm{~s}$ and the profiles for a given initial ciprofloxacin $(3 \mu \mathrm{mol} / \mathrm{ml})$ were reconstructed. Such profiles were used to calculate the amount of ciprofloxacin $(\mu \mathrm{mol})$ transported trough membrane in function of time $(N(t))$. The amount of antibiotic inside the biofilm after time $t$ was calculated according to:

$$
N(t)=s \int_{\mathbf{O}}^{l} C_{m}(x, t) d x,
$$

Where $S$ and $l$ denote respectively the surface $\left(7.0 \times 10^{-5} \mathrm{~m}^{2}\right)$ and thickness of membrane with biofilm, $C_{m}(x, t)$ is the concentration profile inside the membrane with formed biofilm. We assumed the linear concentration distribution (Figure 1C, denotes dashed line):

$$
C_{m}(x, t)=\frac{C_{2}(l, t)-C_{1}(0, t)}{l} x,
$$

Where $C_{1}(0, t), C_{2}(l, t)$ are the concentration values on both surfaces of biofilm membrane. $C_{1}(0, t), C_{2}(l, t)$ and $l$ were determined interferometrically.

\section{Data analysis}

The data were analysed using the Statistica (StatSoft, Tulsa, OK, USA) software package. All the values in this study are expressed as the mean $\pm S D$ from three independent experiments. The differences were compared by the ANOVA test.

\section{Results}

\section{Analysis of PAO1 biofilm formation by $\mathrm{CV}$ staining}

Figure 1 shows the results of $P$. aeruginosa PAO1 biofilm formation for $24-96 \mathrm{~h}$ at $37^{\circ} \mathrm{C}$ in $\mathrm{LB}$ medium under stationary conditions in microtitre plates. The spectrophotometric analysis (CV staining) as well as imageJ software analysis (Figure 2) of plates and nucleopore membranes, respectively, shown that the biofilm is most efficiently formed after $72 \mathrm{~h}$. Moreover, imageJ analysis was used for determination of $\%$ of membrane covering by biofilm (Figure 3). We concluded that the 126 value of grey level is a cut-off point below which 32291 pixels from total analyzed 36180 (membrane with biofilm after $72 \mathrm{~h}$ image) 

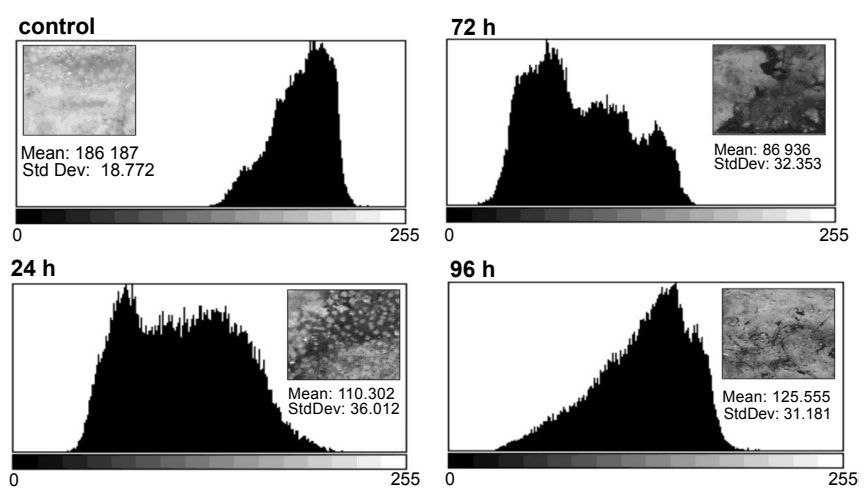

$48 \mathrm{~h}$
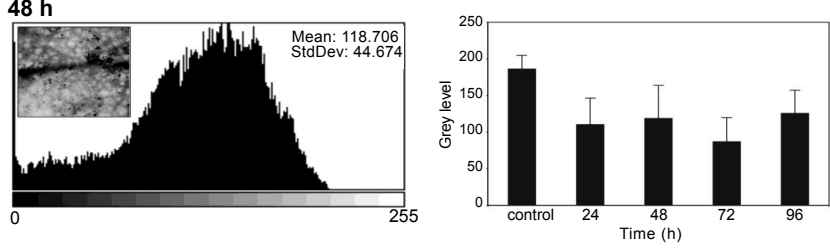

Figure 2: The imageJ analysis (histograms) of nucleopore membrane with pore diameter $0.9 \mu \mathrm{m}$ with $P$. aeruginosa PAO1 biofilm formed for $24-96 \mathrm{~h}$ and stained by crystal violet. Control-bare nucleopore membrane. Bars present the grey level (mean \pm S.D.) of scanned membranes in scale 1-256 measured by imageJ software.

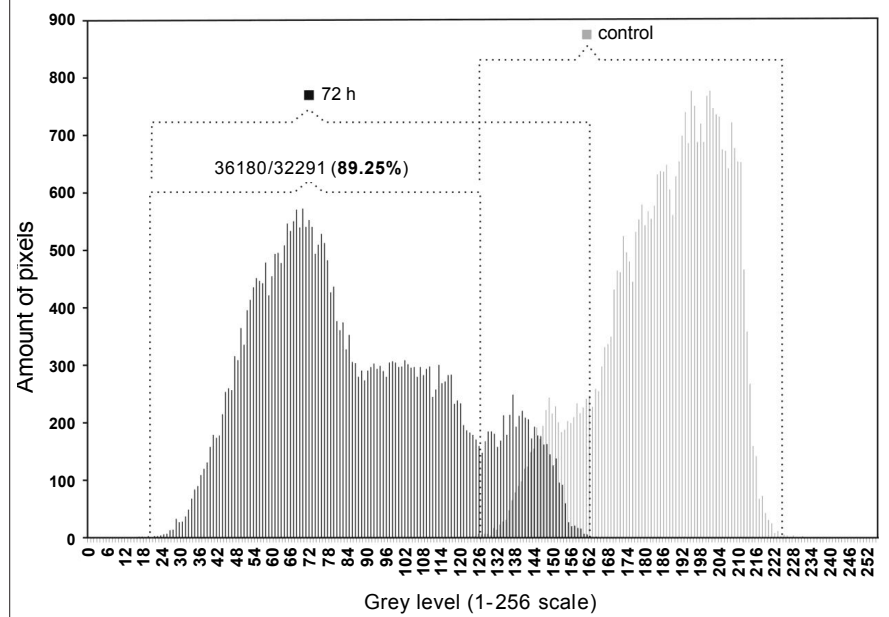

Figure 3: The histograms of imageJ software analysis of bare membrane (control) or with formed biofilm after $72 \mathrm{~h}$ stained by crystal violet. The value 126 of grey level is a cut-off point below which 32291 pixels from total analyzed 36180 (membrane with biofilm after $72 \mathrm{~h}$ image) have the grey level lower than control \% of membrane covering by biofilm is presented.

have the grey level lower than control. It indicates that membrane in $89.25 \%$ is covered by pixels, which grey level is darker that the darkest (126) pixels of bare membrane (control).

\section{Laser interferometry analysis of ciprofloxacin distribution in PAO1 biofilm}

Figure 4 shows experimental set-up of laser interferometry equipment. It consists of a Mach-Zehnder interferometer with a He-Ne laser, a measuring system with membrane (place of biofilm formation), a TV-CCD camera and a computer with a system for the acquisition and processing of interference images. The laser light is spatially filtered and is transformed by the beam expander into a parallel beam ca. 80 mm wide and then split into two beams. The first beam goes through the investigated membrane system with formed bacterial biofilm parallel to the biofilm surface, while the second goes directly through the compensation plate to the light detection system. As a consequence of the superimposition of these beams, respective interference images are generated. The images (interferograms) depend on the refraction coefficient of the solute, which in turn depends on the substance concentration. The concentration of antibiotic transported through biofilm formed on membrane or into biofilm is calculated on the basic of changes of refraction coefficient in function of time.

Figure 5 shows the amount of ciprofloxacin (at the initial concentration of $3 \mu \mathrm{mol} / \mathrm{ml}$ ) transported through biofilm P. aeruginosa PAO1 formed on nucleopore membrane for $72 \mathrm{~h}$. In the control experiments the amount of drug transported through bare membrane increased rapidly for initial times (till $1440 \mathrm{~s}$ ) and then the "plateau effect" was observed when the system reached the steady state. The kinetics of antibiotic diffusion through membrane covered by biofilm had a steady character for $2400 \mathrm{~s}$. At the end, the amount of transported ciprofloxacin was at the level of $25.32 \%(0.0723 \mu \mathrm{mol})$ of total amount of drug diffusing across bare membrane $(0.285 \mu \mu \mathrm{mol})$. It indicated that $0.759 \mu \mathrm{mol}$ of ciprofloxacin was transported through biofilm after $2400 \mathrm{~s}$.

Additionally, laser interferometry method was used to calculate the

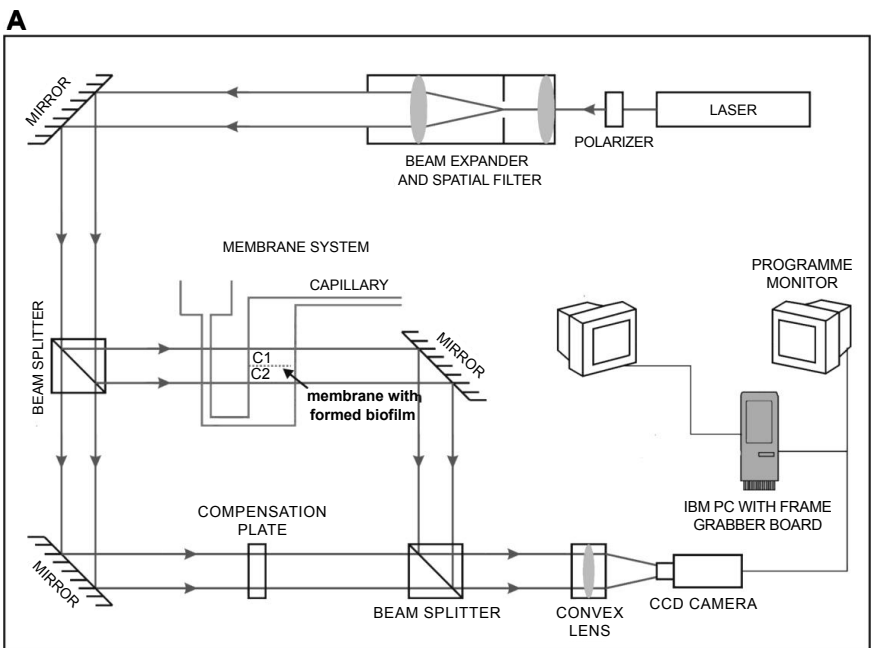

B

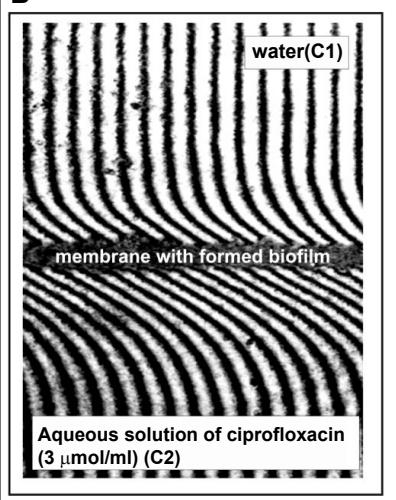

C

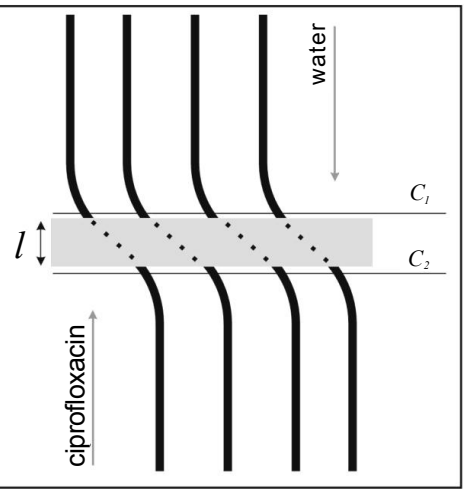

Figure 4: Experimental set-up of the interferometric measurement of ciprofloxacin diffusion through $P$. aeruginosa PAO1 biofilm formed for $72 \mathrm{~h}$ at $37^{\circ} \mathrm{C}(\mathrm{A})$; The interferogram of ciprofloxacin diffusion $(B)$; The method of ciprofloxacin concentration analysis in biofilm structure (C). The description of scheme $A$ is presented in Results section. 


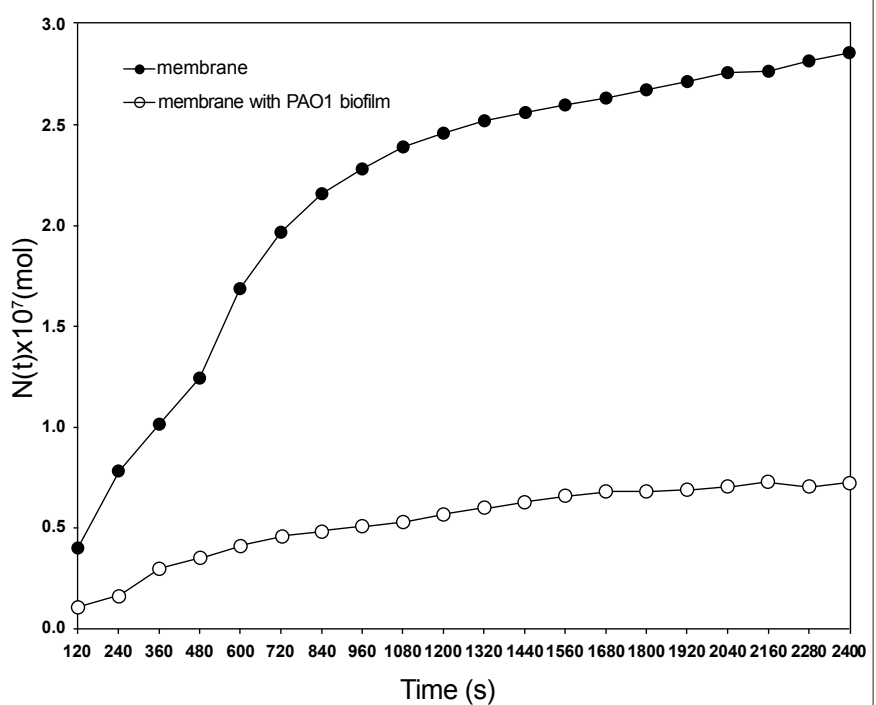

Figure 5: The amount of ciprofloxacin transported through $P$. aeruginosa PAO1 biofilm formed for $72 \mathrm{~h}$ at $37^{\circ} \mathrm{C}$ from initial concentration $3 \mu \mathrm{mol} / \mathrm{ml}$ measured by laser interferometry method

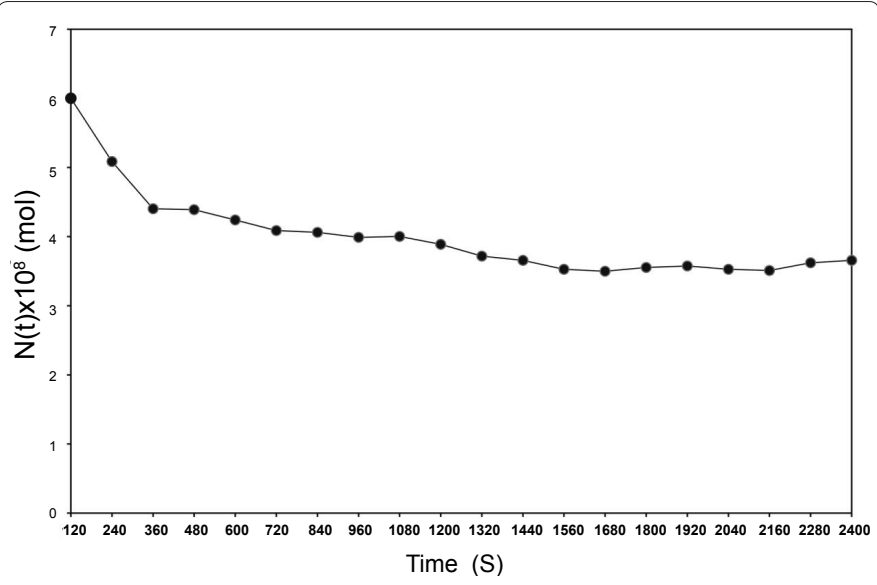

Figure 6: The amount of ciprofloxacin accumulated in $P$. aeruginosa PAO1 biofilm formed for $72 \mathrm{~h}$ at $37^{\circ} \mathrm{C}$ measured by laser interferometry method.

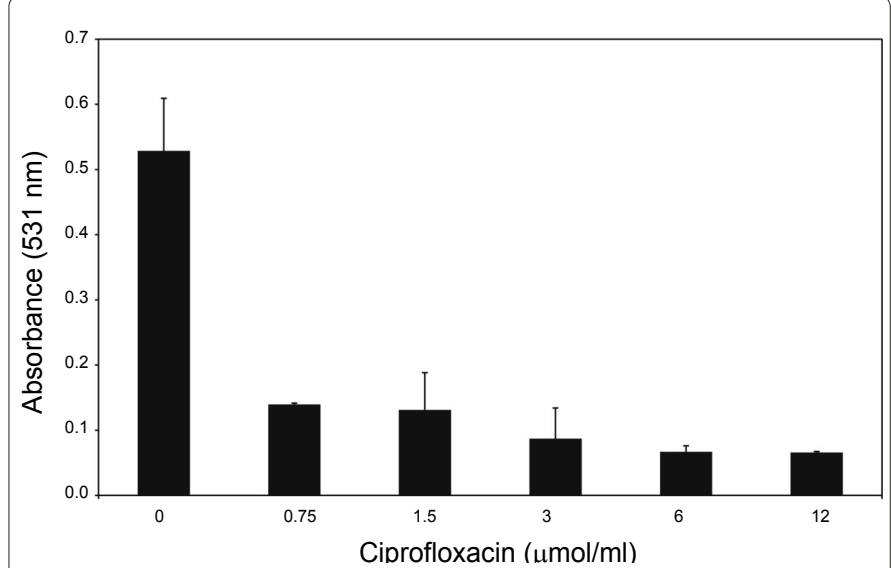

Figure 7: Effects of ciprofloxacin treatment on $P$. aeruginosa PAO1 biofilm measured by crystal violet absorption. amount (mol) of ciprofloxacin accumulated in the P. aeruginosa biofilm structure during the diffusion process. Figure 6 shows that the amount of antibiotic decreased from $6.00 \times 10^{-8} \mathrm{~mol}$ to $3.66 \times 10^{-8} \mathrm{~mol}$ after 2400 s. It indicates that the amount of ciprofloxacin into biofilm is 0.366 $\mu \mathrm{mol}$ after $2400 \mathrm{~s}$ ( $12.2 \%$ of initial amount of antibiotic; $3 \mu \mathrm{mol})$.

\section{Effect of ciprofloxacin on PAO1 biofilm}

Figure 7 shows the effects of ciprofloxacin treatment at different concentrations $(0.75-12 \mu \mathrm{mol} / \mathrm{ml})$ for $2400 \mathrm{~s}$ at $37^{\circ} \mathrm{C}$ on $P$. aeruginosa $\mathrm{PAO} 1$ biofilm. The concentrations higher than $0.75 \mu \mathrm{mol} / \mathrm{ml}$ reduced the mass of biofilm statistically significant $(\mathrm{p}<0.001)$.

\section{Discussion}

P. aeruginosa causes chronic lung infection. Persistent microbial infection by $P$. aeruginosa is the major cause of morbidity and mortality in patients with cystic fibrosis (CF). P. aeruginosa strains isolated from CF patients with advanced stages of disease are distinctive because about $85 \%$ have mucoid colony morphology (overproduction of the EPS alginate and $\mathrm{O}$-acetylated linear polymer of D-mannuronate and L-guluronate). It suggests that mucoid $P$. aeruginosa cells have a distinct survival advantage in the CF lung environment [15]. So, analysis of drug distributions in mucoid structure formed by P. aeruginosa strains is crucial for effective clinical practice directed to bacteria eradication. In this study the laser interferometry method was applied to determine the antibiotic (ciprofloxacin) diffusion efficacy through $P$. aeruginosa biofilm structure.

Ciprofloxacin is an antibacterial agent of the 4-quinolone group derived by systematic modification of the nalidixic acid. The antibacterial properties of ciprofloxacin are associated with (i) inhibition of the intracellular enzymes (DNA-gyrase, topoisomerase IV) and (ii) the efficient transport of antibiotic molecules through bacterial envelopes and cytoplasmic membranes [16,17]. The effective transport of ciprofloxacin through biofilm is strongly associated with its biophysical properties. This drug formed four different microspecies molecules (neutral, zwitterion, positively and negatively charged) depending on the $\mathrm{pH}$ of the solution and this diversity promotes efficient diffusion through bacterial biofilm [17].

Using ciprofloxacin as model antibiotic, we showed that $25.32 \%$ of $3 \mu \mathrm{mol}$ antibiotic solution was transported through PAO1 biofilm (Figure 5) and the amount of ciprofloxacin accumulated in the biofilm was $12.2 \%$ of initial drug amount after $2400 \mathrm{~s}$ of treatment (Figure 6). Ciprofloxacin in this initial amount $(3 \mu \mathrm{mol})$ was sufficient to PAO1 biofilm eradication (Figure 7).

In conclusion, laser interferometry analysis of drug distribution though bacterial biofilm might be useful in modeling of antibiotics diffusion in correlation with their biological effects. The practical application of laser interferometry might be associated with antibacterial efficacy tests especially used for biofilm eradication. Two elements can be determined using interferometry: (i) quantitative analysis of drug diffusion through biofilm, and (ii) drugs distribution in biofilm structure.

\section{Acknowledgements}

This work was supported by grant 2012/04/M/NZ6/00335 from the National Science Centre, Poland. We would like to thank Magdalena Zych and Wioletta Łakomiec for technical support. Michal Arabski and Zuzanna Drulis-Kawa acknowledge project COST BM1003 "Microbial cell surface determinants of virulence as targets for new therapeutics in Cystic Fibrosis". 
Citation: Arabski M, Wąsik S, Drulis-Kawa Z (2013) Laser Interferometry Analysis of Ciprofloxacin Diffusion through Pseudomonas aeruginosa Biofilm. Clin Microbial 2: 105. doi:10.4172/2327-5073.1000105

\section{References}

1. Mah TF, O'Toole GA (2001) Mechanisms of biofilm resistance to antimicrobial agents. Trends Microbiol 9: 34-39.

2. Anderl JN, Franklin MJ, Stewart PS (2000) Role of antibiotic penetration limitation in Klebsiella pneumoniae biofilm resistance to ampicillin and ciprofloxacin. Antimicrob Agents Chemother 44: 1818-1824

3. Costerton JW, Stewart PS, Greenberg EP (1999) Bacterial biofilms: a common cause of persistent infections. Science 284: 1318-1322.

4. Evans DJ, Allison DG, Brown MR, Gilbert P (1991) Susceptibility of Pseudomonas aeruginosa and Escherichia coli biofilms towards ciprofloxacin: effect of specific growth rate. J Antimicrob Chemother 27: 177-184.

5. Shigeta M, Tanaka G, Komatsuzawa H, Sugai M, Suginaka H, et al. (1997) Permeation of antimicrobial agents through Pseudomonas aeruginosa biofilms: a simple method. Chemotherapy 43: 340-345.

6. Suci PA, Mittelman MW, Yu FP, Geesey GG (1994) Investigation of ciprofloxacin penetration into Pseudomonas aeruginosa biofilms. Antimicrob Agents Chemother 38: 2125-2133.

7. Arabski M, Wasik S, Dworecki K, Kaca W (2007) Laser interferometric determination of ampicillin and colistin transfer through cellulose biomembrane in the presence of Proteus vulgaris O25 lipopolysaccharide. J Memb Sci 299: 268-275.

8. Arabski M, Davydova VN, Wasik S, Reunov AV, Lapshina LA, et al. (2009) Binding and biological properties of lipopolysaccharide from Proteus vulgaris O25 (48/57) with chitosan. Carbohydrate Polymers 78: 481-487.

9. Arabski M, Wasik S, Dworecki K, Kaca W (2009) Laser interferometric and cultivation methods for measurement of colistin/ampicilin and saponin interactions with smooth and rough of Proteus mirabilis lipopolysaccharides and cells. J Microbiol Methods 77: 179-183.

10. Arabski M, Wasik S, Piskulak P, Góźdź N, Slezak A, Kaca W, et al. (2011) Analysis of antibiotic diffusion from agarose gel by spectrophotometry and laser interferometry methods. Polim Med 41: 25-32.

11. Arabski M, Wasik S, Grzeskiewicz H, Drulis-Kawa Z, Gubernator J, et al (2012) Laser interferometric determination of liposome diffusion through artificial membranes: Interferometry-Research and Applications in Science and Technology. INTECH.

12. Gula G, Waszczuk K, Olszak T, Majewska J, Gotszalk T, et al. (2012) Piezoelectric tuning fork based mass measurement method as a novel tool for determination of antibiotic activity on bacterial biofilm. Sensors \& Actuators: B. Chemical 175: 34-39.

13. Schneider CA, Rasband WS, Eliceiri KW (2012) NIH Image to ImageJ: 25 years of image analysis. Nat Methods 9: 671-675.

14. Arabski M, Konieczna I, Sołowiej D, Rogoń A, Kolesińska B, et al. (2010) Are anti-Helicobacter pylori urease antibodies involved in atherosclerotic diseases? Clin Biochem 43: 115-123.

15. Stapper AP, Narasimhan G, Ohman DE, Barakat J, Hentzer M, et al. (2004 Alginate production affects Pseudomonas aeruginosa biofilm development and architecture, but is not essential for biofilm formation. J Med Microbiol 53: 679-690.

16. Weigel LM, Steward CD, Tenover FC (1998) gyrA mutations associated with fluoroquinolone resistance in eight species of Enterobacteriaceae. Antimicrob Agents Chemother 42: 2661-2667.

17. Hernández-Borrell J, Montero MT (2003) Does ciprofloxacin interact with neutral bilayers? An aspect related to its antimicrobial activity. Int J Pharm 252 149-157. 